Www.jmscr.igmpublication.org

Impact Factor (SJIF): 6.379

Index Copernicus Value: 79.54

ISSN (e)-2347-176x ISSN (p) 2455-0450

crossrefDOI: https://dx.doi.org/10.18535/jmscr/v6i10.19

Journal Of Medical Science And Clinical Research

IGM Publication

An Official Publication of IGM Publication

\title{
A Study on Etiopathology and predictors of Erythema Nodosum in a Tertiary care hospital of West Bengal
}

\author{
Authors: \\ Dr Atanu Chandra', Dr Indranil Sen ${ }^{2}$, Dr Babulal Daulagajao ${ }^{3}$, Dr Saikot Ganai ${ }^{4}$
}

Medical College Kolkata, 88 College Street, College Square, Kolkata West Bengal Pin-700073

Corresponding Author

Dr Indranil Sen

Address: HB-8/2 Sector-3 Salt Lake Kolkata Pin: 700106, India

Email: docindranilsen@gmail.com, Phone Number: 8902725184

\begin{abstract}
Aim: To study the etiology of Erythema nodosum (EN) and co-morbidities associated with it among a subset of Indian patients, clinical variation between idiopathic and secondary EN and also to assess the factors predictive for the development of EN.

Method: A cross sectional study was done on thirty patients with EN diagnosed on clinical basis. Patients were subjected to clinical examination, laboratory \& radiological investigations including histopathology. Etiology of EN \& associated co-morbidities were evaluated. Also the clinical variation between idiopathic and secondary EN was assessed.

Result: The frequency of unknown cause(Idiopathic EN) in our study was56.7\%.The etiology could be determined in $43.3 \%$ of cases:- Streptococcal infection (23.1\%), Tuberculosi s(15.4\%), Drugs (15.4\%), Pregnancy (15.4\%), Behcet's disease (15.4\%), Sarcoidosis (7.7\%), Inflammatory bowel disease (7.7\%). $83.3 \%$ of the total patients were female \& the mean age was 28.18 years in the idiopathic EN and 27.23 years in secondary EN. Hypothyroidism was significantly associated with idiopathic EN. We found significant differences between the idiopathic \& secondary causes regarding history of fever within last 6 weeks, arthritis, cough, chest X ray abnormalities \& ASO titre.

Conclusions: Etiological diagnosis was possible in $43.3 \%$ of cases. Most common cause was Streptococcal infection. Most of the cases were female. Idiopathic EN was significantly associated with hypothyroidism. Features like history of fever within last 6 weeks, arthritis, cough, chest $X$ ray abnormalities \&ASO titre were predictors of secondary EN. We compared our study results with other studies \& emphasize the need of a detailed workup for all patients of EN because it's harbinger of common and treatable conditions.

Keywords: Erythema nodosum, Tuberculosis, Sarcoidosis, Behcet's disease.
\end{abstract}

\section{Introduction}

Erythema nodosum (EN) is generally considered as a benign and self-limiting hypersensitivity reaction which may be idiopathic or associated with benign infections, sarcoidosis, rheumatologic disease, medications, inflammatory bowel disease, autoimmune disorder, pregnancy and some malignancies. $^{1,2}$ It is the most common 
clinicopathological variant of septal pannicullitis usually first evident as outcropping of erythematous tender nodule located predominantly over the extensor aspect of the lower extremities. Panniculitis is characterized by inflammatory changes affecting primarily the subcutaneous adipose tissue which can become granulomatous in the histiocytic phase. ${ }^{3,4}$ The usual clinical presentation of EN is sudden onset appearance of symmetrical, tender, erythematous, warm nodules usually located in the extensor surfaces on the shins, ankle and knees which are bilaterally distributed most often. Ulceration of the nodules is unusual and they heal without atrophy or scaring. It is often preceded by non-specific prodromal symptoms of 1-3 weeks, which may include fever, malaise and symptoms of upper respiratory tract infection. It is usually selflimiting and last for two to six weeks. Diagnosis of EN is mainly based on clinical features, but in doubtful cases biopsy of the lesion may be helpful. 5, 6, 7, 8 Treatment of erythema nodosum should be directed to the underlying associated condition, if identified. Usually, nodules of EN regress spontaneously within a few weeks, and bed rest is often sufficient treatment. Aspirin and non steroidal anti-inflammatory drugs may be helpful to enhance analgesia and resolution. ${ }^{9}$

$\mathrm{EN}$ is not a very uncommon condition and often it is associated with some underlying secondary condition, which remain undiagnosed if no proper evaluation is done. Even idiopathic erythema nodosum may be over investigated in some cases. The objective of the present study was to identify the underlying cause and associated co morbidities in case of $\mathrm{EN}$ and to evaluate the clinical presentation and variations in idiopathic and secondary EN.

\section{Materials and Methods}

Total 30 patients of EN attending Rheumatology, Dermatology and Medicine outpatient department in a tertiary care hospital of Kolkata from December 2013 to December 2014 were included in our study. Patients presenting with painful \& reddish subcutaneous nodules located on anterior aspect of the legs or any other sites (In some doubtful cases, histopathology showing septal panniculitis without vasculitis) who gave informed consent were taken as cases. Panniculitis associated with ulceration, atrophy or local infections were excluded from our study. Etiology of Erythema nodosum and co-morbidities associated with it and clinical variation between idiopathic and secondary EN was evaluated in our cross-sectional, descriptive, observational study. The evaluation of erythema nodosum in these patients was done on the basis of history taking, thorough clinical examination, laboratory investigations (like complete haemogram, ESR, ASO titre, serum ACE), radiological imaging (Chest X ray \& CT Scan chest in certain cases), Mantoux test, Sputum for AFB \& throat swab for culture if clinically indicated and lastly biopsy from the lesion in doubtful cases. Written informed consent was obtained from the patients and the study protocol was approved by Institutional Ethics committee for human research, Medical College, Kolkata.

\section{Statistical Analysis}

Categorical variables are expressed as Number of patients and percentage of patients and compared across the 2 groups using Pearson's Chi Square test for Independence of Attributes.

Continuous variables are expressed as Mean \pm Standard Deviation and compared across the 2 groups using Mann-Whitney U test since the data does not follow normal distribution.

The statistical software SPSS version 20 has been used for the analysis.

An alpha level of 5\% has been taken, i.e. if any $\mathrm{P}$ value is less than 0.05 it has been considered as significant.

\section{Results}

Total 30 patients of erythema nodosum were included in our study. Those 30 cases of EN (25 Females) diagnosed based on clinical findings was taken, evaluated and compared between known 
cause of EN (secondary EN) and unknown cause (idiopathic EN).Other 8 cases of panniculitis associated with ulceration, atrophy or local infection were excluded from our study. Among all cases of EN, most ( 25 patients) were female (83.33\%). Most of the patients (27 patients) were younger than $40 \mathrm{yrs}(90 \%) \&$ only 3 patients out of $30(10 \%)$ were in the age group of 41 to 60 years. After detailed clinical examination and laboratory investigation we have found the etiological factor of EN in some cases. Among all cases, 17 patients $(56.7 \%$ of the cases) were idiopathic and 13 patients (43\% of the cases) were secondary EN in our study.

Among the secondary causes EN, 3 patients ( $23.1 \%$ of all the secondary causes of EN) were post streptococcal in etiology as evidenced by growth of streptococci in culture of throat swab in clinically indicated cases; Tuberculosis, pregnancy, Drugs \& Behcet's disease was the etiology in $15.4 \%$ cases in each group (2 patients out of 13 in each); others included one case $(7.7 \%$ all the secondary causes of EN) of Sarcoidosis \& inflammatory bowel disease.

We have also searched for associated co morbidities of EN other than etiologies. We have found 4 cases were associated with hypothyroidism \& more interestingly all the 4 cases of hypothyroidism were in the idiopathic group of EN. There was presence of hypertension, diabetes, Ankylosing Spondylitis and chronic pancreatitis one each in idiopathic EN. In secondary EN we have found one case of Wilson disease and HIV.

EN can occur at any site. However the most commonly involved sites are over shins, knee and ankle. The sites of involvement of EN in our study group are as follows: only shin in 11 patients out of 30 cases of $\mathrm{EN}(36.67 \%)$; shin \& calves in 9 patients (30\%); shin, calves \& other sites in 8 patients $(26.67 \%) \&$ other sites (upper extremities) in only 2 cases $(6.67 \%)$.

We have also evaluated a detailed comparison of different clinical, laboratory \& radiological parameters between the known cause of EN (secondary EN) and unknown cause (idiopathic $\mathrm{EN})$.

Clinical, laboratory \& radiological parameters between the known cause of EN (secondary EN) and unknown cause (idiopathic EN) are depicted in Table1 \& Table2.

Though EN is predominantly seen in females, there was no significant difference between the idiopathic and secondary groups. Each of the features like history of sore throat within last 6 weeks, arthritis, cough \& chest $X$ ray abnormalities in the form of patchy opacities and hilar adenopathy were present in 3 patients of secondary $\mathrm{EN}$, whereas they were present in none of the idiopathic cases. In our study, we had found significantly increased occurrence of features like history of sore throat within last 6 weeks, arthritis, cough \& chest $\mathrm{X}$ ray abnormalities in patients of secondary EN in comparison with the idiopathic group. There were no significant difference between those two groups with respect to features like sex, history of fever within last 6 weeks, fatigability, arthralgia, diarrhoea, having past history of EN , presence of multiple lesions ( $>5$ in number) or Positive Mantoux test (induration of $>10 \mathrm{~mm}$ ).

There were significantly increased values of ASO titre in the secondary group, though there was no difference in these two groups with respect to ESR $\&$ leukocytosis. 
Table 1: Comparison of clinical \& radiological features of EN between Idiopathic \& Secondary Causes

\begin{tabular}{|l|c|c|c|}
\hline \multirow{2}{*}{ FEATURES } & \multicolumn{2}{|c|}{ TYPES OF EN } & \multirow{2}{*}{ P value } \\
\cline { 2 - 3 } & $\begin{array}{c}\text { IDIOPATHIC } \\
(\mathrm{n}=17)[\%]\end{array}$ & $\begin{array}{c}\text { SECONDARY } \\
(\mathrm{n}=13)[\%]\end{array}$ & \\
\hline FEMALE SEX & $15[88]$ & $10[77]$ & 0.410 \\
FEVER WITHIN 6 WEEKS & $13[76]$ & $9[69]$ & 0.657 \\
FATIGUE & $10[59]$ & $7[54]$ & 0.785 \\
H/O SORE THROAT<6 WEEKS & $0[0]$ & $3[23]$ & $\mathbf{0 . 0 3 7}$ \\
ARTHRALGIA & $6[35]$ & $4[31]$ & 0.794 \\
ARTHRITIS & $0[0]$ & $3[23]$ & $\mathbf{0 . 0 3 7}$ \\
COUGH & $0[0]$ & $3[23]$ & $\mathbf{0 . 0 3 7}$ \\
DIARHOEA & $0[0]$ & $1[8]$ & 0.240 \\
PAST H/O EN & $2[12]$ & $0[0]$ & 0.201 \\
MULTIPLE LESIONS(>5) & $7[41]$ & $3[23]$ & 0.297 \\
X RAY ABNORALITIES & $0[0]$ & $3[23]$ & $\mathbf{0 . 0 3 7}$ \\
POS. MANTOUX TEST(>10mm) & $2[12]$ & $3[23]$ & 0.410 \\
ASSOCIATED HYPOTHYROIDISM & $4[24]$ & $0[0]$ & $\mathbf{0 . 0 2 2}$ \\
\hline
\end{tabular}

Table 2: Comparison of age \& Laboratory parameters between Idiopathic \& Secondary EN

\begin{tabular}{|c|c|c|c|}
\hline \multirow{2}{*}{ PARAMETERS } & \multicolumn{2}{|c|}{ TYPES OF EN } & \multirow{2}{*}{ p VALUE } \\
\cline { 2 - 3 } & IDIOPATHIC & SECONDARY & \\
\cline { 2 - 3 } & Mean \pm Std. Deviation & Mean \pm Std. Deviation & \\
\hline AGE & $29.94 \pm 12.06$ & $27.23 \pm 7.88$ & 0.516 \\
TLC & $8784.88 \pm 3048.58$ & $8403.15 \pm 2168.87$ & 0.786 \\
ESR & $52.65 \pm 12.09$ & $44.85 \pm 10.38$ & 0.062 \\
ASO TITRE & $155.29 \pm 27.29$ & $252.75 \pm 81.57$ & $\mathbf{0 . 0 4 3}$ \\
\hline
\end{tabular}

\section{Discussion}

In our study, total 30 cases of clinically diagnosed EN were evaluated and a detailed comparison was done on the basis of clinical and laboratory parameters between the known cause of EN (secondary EN) and unknown cause (idiopathic EN). Our main objectives were to identify the etiology of $\mathrm{EN}$, to find out any associated co morbidities and the predictors of the secondary EN.

In our study, we have found male to female ratio of 1:5.The mean age of the patients was 28.18 years in case of idiopathic and 27.23 years in secondary EN in both the genders. This is in accordance with the previous studies which demonstrated that EN occurs three to six times more frequently in women than men, although the sex ratio is approximately equal before the pubertal age group. ${ }^{10}$ It has been reported that EN occurs between the second and fourth decades of life, with the peak incidence between 20 and 30 years of age, probably because of the high incidence of sarcoidosis at this age. ${ }^{6,7,11}$
The diagnosis of EN can usually be made clinically, with no need for histopathological examination for the diagnosis. With the exception of three cases, all our patients were diagnosed with EN based upon clinical findings.

The legs were the most common sites of involvement and although usually bilateral, lesions can also be unilateral. In this study, eight (26.3\%) patients had EN located on the upper as well as lower extremities.

Erythema nodosum has been associated with a variety of infectious diseases, most notably tuberculosis, streptococcal infections, Yersinia enterocolitica, Y. pseudotuberculosis, cat-scratch disease, tularemia, acute upper respiratory tract infections, Q fever, and epidemiologic internal organ mycoses (histoplasmosis, coccidiomycosis etc). ${ }^{12,13,14,15,16}$ Chronic inflammatory conditions includes Behcet's disease, Reiter disease, systemic lupus erythematosus, inflammatory bowel disease, and sarcoidosis, malignant diseases, and exposure to certain medications can be involved in the etiology of EN. ${ }^{17,18,19,20,21,22}$ Streptococcal infection was reported as the most common cause 
of EN in the pediatric as well as adult population.

${ }^{12}$ In our study group we have found streptococcal and tubercular as the most common cause of secondary EN (23.1\%)

A study done by Aydin-Teke $\mathrm{T}$ et al studied 39 cases of EN in Turkey where they have found $23 \%$ of cases were due to streptococcal infection. ${ }^{23}$ Whereas Mert A et al had evaluated 100 cases of EN in Istanbul where they have found $11 \%$ of $\mathrm{EN}$ were due to streptococcal infections. $^{24}$ So geographical variation leads to different percentage of streptococcal infection leading to EN. However streptococcal infection is considered as the most cause of secondary EN worldwide. ${ }^{8,25,26,27}$

The second most common etiologic factors in our study were tuberculosis. It has been reported that EN can be the first and only presentation of tuberculosis in a patient with/without evidence of active disease. ${ }^{28}$ Tuberculosis should be investigated and excluded, especially in endemic areas, as an underlying etiology of EN.

Sarveswari K N et al had evaluated 15 cases of EN, in Anna Nagar, Chennai. And found 7 cases were of tubercular, which indicates that tuberculosis is a common cause of $\mathrm{EN}$ in our country where there is high prevalence of tuberculosis. $^{29}$

Behcet's disease is also an important etiology of EN. For the diagnosis, the criteria of International Study Group for Behçet's Disease are used. In our study group we had found 2 Behcet's disease presenting with EN comprising of $15 \%$ of the secondary cases.Mert A et al had evaluated 100 cases of EN in which Behçet's Disease was found as the etiology of EN in $6 \%$ of our cases according to the criteria of International Study Group. ${ }^{30}$

Although many drugs may lead to EN like oral contraceptives, antibiotics, aspirin, non-steroid anti-inflammatory drugs etc. In our study we have got 2 patients, among the EN series, drugs have been the fourth most frequent etiology comprising $15.4 \%$. one patient develops EN after intake of OCP and in one HIV patient EN develop after 3 days of intake of sulfonamides. Literature had reported HIV itself as a cause of EN. However literature had reported 3 to $15 \%$ cases of EN are caused by drugs. ${ }^{30,31,32}$

Among the secondary EN in our study group, we had found only one case of EN in pregnancy, sarcoidosis and ulcerative colitis (comprising 7\% each). Erythema nodosum is associated with sarcoidosis in 10 to $35 \%$ of cases. ${ }^{8,11}$ Psychos DN et al in one prospective study in Greece found that out of 132 cases of erythema nodosum $28 \%$ was associated with sarcoidosis. ${ }^{27}$ Mert A et al had evaluated 100 cases of EN in Istanbul where they had found $10 \%$ of cases were associated with sarcoidosis. ${ }^{30}$ There is variation according to geographical distribution. We have found less may be as because it has reported that sarcoidosis is less in prevalence in South East Asian countries.

EN is associated with wide variety of infection literature has been reported even with viral infection like CMV and herpes simplex. We have not found in any of our cases it may as we have not done PCR for viral infection due to limited resource in our study population.

Despite thorough clinical examination and extensive laboratory investigation we have found etiology of EN in $43.3 \%$ of cases and $56.7 \%$ we could not find any etiology in our study population. It has reported between $17 \%$ and $72 \%$ of cases are idiopathic., ${ }^{5,6,30}$ Our study also corresponds to the literature.

Although not very frequent, EN is encountered all over the world and etiological description rests upon both medical knowledge and experience .To differentiate between primary and secondary cases maybe a challenge for the physician. Since there are plenty of probable etiologies, a wisely devised and cost-effective diagnosis is crucial. Generally, the etiology should be searched by a basic clinical approach .Uncovering the etiology could not be possible in nearly half of the cases. Since the definite recognition of the etiologies are challenging when a patient is seen with EN for the first time, it is critical to know the factors predicting secondary EN. In our study, we had 
found significantly increased occurrence of features like history of sore throat within last 6 weeks, arthritis, cough, chest $\mathrm{X}$ ray abnormalities \& ASO titer in patients of secondary EN in comparison with the idiopathic group. There were no significant difference between those two groups with respect to features like sex, history of fever within last 6 weeks, fatiguability, arthralgia, diarrhea, having past history of EN, presence of multiple lesions ( $>5$ in number) or Positive Mantoux test (induration of $>10 \mathrm{~mm}$ ). Our study is mostly in accordance with the previous studies except a few predictors of secondary EN. This may be due to racial, geographic, environmental differences $\&$ the relatively smaller sample size of our study.

Among the five main EN series published in last 40 years, a study conducted by Garcia-Porrua $\mathrm{C}$ et al found chest x-ray abnormalities, history of non-streptococcal upper respiratory tract infections, higher ASO level, tuberculin skin test, presence of synovitis and diarrhea were predictors of secondary EN. ${ }^{26}$ A study conducted by A. Mert et al on 100 cases of EN in Istanbul found that the clinical factors favouring secondary EN are fever, leukocytosis, elevated CRP level, accelerated ESR, presence of cough, sore throat, diarrhea, arthritis, and pulmonary pathology. ${ }^{30}$

Another interesting finding we had found in our study that 4 patients of idiopathic EN was hypothyroid whereas there was no hypothyroid in the secondary group. This is not mentioned in any of the previous literatures. This may be due to the fact that hypothyroidism is a very common problem in our country, our study is based on relatively smaller sample size or there may be an unknown autoimmune mechanism responsible for both of these conditions.

Therefore, all patients of erythema nodosum need detailed evaluation to find out any underlying systemic disease. Further Indian studies may be needed on this topic to find out etiological factors \& predictors EN.

\section{References}

1. Bondi EE, Margolis DJ, Lazarus ZS. Panniculitis. In: Freedberg I, Eisen A, Wolff K, et al, eds. Fitzpatrick's dermatology in general medicine. 5th ed. New York, NY: McGraw-Hill; 1999: 1284-1286.

2. Requena L, Requena C. Erythema nodosum (review). Dermatol Online J. 2002; 8:4.

3. Schwartz RA, Nervi SJ. Erythema nodosum: a sign of systemic disease. Am Fam Physician. 2007; 75:695-700. [PubMed]

4. Blake T, Manahan M, Rodins K. Erythema nodosum - a review of an uncommon panniculitis. Dermatol Online J. 2014; 20:22376. [PubMed]

5. Acosta KA, Haver MC, Kelly B. Etiology and therapeutic management of erythema nodosum during pregnancy: an update. Am J Clin Dermatol.2013; 14:215-220. [PubMed]

6. Bohn S, Buchner S, Itin, P. Erythema nodosum: 112 cases. Epidemiology, clinical aspects and histopathology. Swiss Med Weekly. 1997; 127(27-28): 11681176. [PMID 9324]

7. Requena L, SanchezYus E. Erythema nodosum. Dermatol Clin.2008; 26: 425438. [PMID 18793974]

8. Cribier B, Caille A, Heid E, Grosshans E.Erythema nodosum and associated diseases. A Study of 129 cases. Int J Dermatol.1998; 37:667-72.

9. Friedman ES, LaNatra N, Stiller MJ. NSAIDs in dermatologic therapy: review and preview. J Cutan Med Surg. 2002; 6:449-459.[Abstract]

10. Jørgensen KT, Pedersen BV, Nielsen NM, Jacobsen S, Frisch M. Childbirths and risk of female predominant and other autoimmune diseases in a populationbased Danish cohort. J Autoimmun. 2012; 38:J81-7. [PubMed] 
11. Costabel U, Guzman J, Drent $M$. Diagnostic approach to sarcoidosis. Eur Respir Monograph. 2005; 10:259-260.

12. Uhl JR, Adamson SC, Vetter EA, Schleck $\mathrm{CD}$, Harmsen WS, Iverson $\mathrm{LK}$, et al. Comparison of LightCycler PCR, rapid antigen immunoassay, and culture for detection of group A streptococci from throat swabs. J Clin Microbiol. 2003; 41:242-9.

13. Mert A, Ozaras R, Tabak F, Ozturk R. Primary tuberculosis cases presenting with erythema nodosum. J Dermatol. 2004;31:66-8.

14. Louthrenoo W, Lertprasertsuke N, Kasitanon N, Sukitawut W. Erythema nodosum as a manifestation of HIV infection. Asian Pac J Allergy Immunol. 2002; 20:175-8.

15. Sanders CJ, Hulsmans RF. Persistent erythema nodosum and asymptomatic Campylobacte rinfection. $\mathrm{J}$ Am Acad Dermatol. 1991; 24(2 pt 1)285-6.

16. Bottone EJ. Yersinia enterocolitica: the charisma continues. Clin Microbiol Rev. 1997;10:257-76.

17. Gupta M, Singh K, Lehl SS, Bhalla M. Recurrent erythema nodosum: a red flag sign of hidden systemic vasculitis. BMJ Case Rep. 2013;2013 doi: 10.1136/bcr2013-009507. [PMC free article] [PubMed][Cross Ref]

18. Misago N, Tada Y, Koarada S, Narisawa Y. Erythema nodosum-like lesions in Behçet's disease: a clinicopathological study of 26 cases. Acta Derm Venereol. 2012;92:681-686. [PubMed]

19. Huang BL, Chandra S, Shih DQ. Skin manifestations of inflammatory bowel disease. Front Physiol. 2012;3:1-6.

20. Racanelli V, Prete M, Minoia C, et al. Rheumatic disorders as paraneoplastic syndromes. Autoimmun Rev.2008;7:352358. [PubMed]
21. Trost L, McDonnell J. Important cutaneous manifestations of inflammatory bowel disease. Postgrad Med J. 2005;81:580-585.[Abstract]

22. Yang SG, Han KH, Cho KH, Lee AY. Development of erythema nodosum in the course of oestrogen replacement therapy. Br J Dermatol. 1997;137:319-20.

23. Aydın-Teke $\quad T^{1}$, Tanır G, Bayhan GI, Metin O, Oz N. Erythema nodosum in children: evaluation of 39 patients. Turk $\mathbf{J}$ Pediatr. 2014 Mar-Apr;56(2):144-9

24. Mert A, Ozaras R, Tabak F, Pekmezci S, Demirkesen C, Ozturk R. Erythema nodosum: an experience of 10 years. Scand J Infect Dis. 2004;36:424-7.

25. Erez A, Horowitz J, Sukenik S. Erythema nodosum in the Negev area - a survey of 50 patients. Isr J Med Sci. 1987; 23(12):1228. [PMID 34407]

26. García-Porrúa $C^{1}$, González-Gay MA, Vázquez-Caruncho M, López-Lazaro L, Lueiro M, Fernández ML, AlvarezFerreira J, Pujol RM. Erythema nodosum: etiologic and predictive factors in a defined population. Arthritis Rheum. 2000 Mar;43(3):584-92

27. Psychos DN, VoulgarisPV, Skopouli FN, DrosoS AA, Moutsopoulos HM.Erythema nodosum:the underlying condition. Clin Rheumatol 2000:19 212-6.

28. Whig J, Mahajan V, Kashyap A, Gupta S. Erythema nodosum: atypical presentation of common disease.Lung India 2010;27: 181-182.

29. Sarveswari K N, Swamikannu M, Ramakrishnan S. An aetiological analysis of erythema nodosum in a community hospital. Indian J Dermatol Venereol Leprol 2001; 67:287-9

30. Mert A, Kumbasar H, Ozaras R, et al. Erythema nodosum: an evaluation of 100 cases. Clin Exp Rheumatol. 2007; 25:56370. [PubMed] 
31. Kakourou T,Drosatou P, Psychou F, Aroni $\mathrm{K}$, Nicholaidou p,.Erythema nodosum in children.J Am acad Dermatol 2011;44:1721.

32. Fox MD, Schwartz RA. Erythema nodosum. Am Fam Physician 1992; 46:818-22. 